\title{
Características das vítimas por acidentes de transporte terrestre em município da Região Sul do Brasil
}

Victims' characteristics by road accidents in a city of Southern Brazil

\section{Selma Maffei de Andrade e Maria Helena P de Mello Jorge ${ }^{b}$}

aD epartamento M aterno-infantil e Saúde Comunitária da U niversidade Estadual de Londrina. Londrina, PR, Brasil. ' ${ }^{b}$ epartamento de Epidemiologia da Faculdade de Saúde Pública da U niversidade de São Paulo. São Paulo, SP, Brasil 


\title{
Características das vítimas por acidentes de transporte terrestre em município da Região Sul do Brasil* \\ Victims' characteristics by road accidents in a city of Southern Brazil
}

\author{
Selma Maffei de Andrade ${ }^{a}$ e Maria Helena P de Mello Jorge \\ a Departamento Materno-infantil e Saúde Comunitária da Universidade Estadual de Londrina. Londrina, PR, \\ Brasil. ${ }^{b}$ Departamento de Epidemiologia da Faculdade de Saúde Pública da Universidade de São Paulo.
}

São Paulo, SP, Brasil

\section{Descritores}

Acidentes de trânsito, estatística\# .

Sistemas de informação, utilização\#. Morbidade\#. Mortalidade. Registros hospitalares. Serviços médicos de emergência.

\section{Resumo}

\section{Objetivo}

Avaliar a magnitude da morbimortalidade por acidentes de transporte terrestre e as características das vítimas.

\section{Métodos}

Foram estudadas 3.643 vítimas de acidentes de transporte terrestre ocorridos em Londrina, Paraná, no primeiro semestre de 1996, abrangendo as registradas pela Polícia Militar, as que morreram no local do evento ou no trajeto para o hospital, além das atendidas em serviços de pronto-socorro ou internadas pelo Sistema Único de Saúde. Foi observado um prazo de 180 dias para verificar ocorrência de óbito.

\section{Resultados}

Os coeficientes de incidência de agravos e de mortalidade médios por acidentes de transporte terrestre foram de 1582,2 e 29,0 por 100.000 habitantes, respectivamente. Esses coeficientes, entretanto, mostraram grande variabilidade em relação a diversas características (local de residência, sexo, idade e categoria da vítima). Motociclistas representaram o principal tipo de vítima, seguidos por ciclistas e pedestres, perfazendo, juntos, $76,9 \%$ do total de vítimas e $81,5 \%$ das que morreram.

Conclusões

Os resultados revelam que os acidentes de transporte terrestre constituem-se importante causa de morbimortalidade e sugerem a necessidade de estratégias específicas de prevenção com vistas a reduzir esses eventos, principalmente os que envolvem os usuários mais vulneráveis da via pública.

\section{Abstract}

\section{Objective}

Keywords

Accidents, traffic, statistics ${ }^{\#}$.

Information systems, utilization ${ }^{\#}$. Morbidity.
To evaluate the magnitude of morbidity and mortality and describe some of the characteristics of road accident victims.

Methods

The study population was composed of 3,643 victims of road accidents which occurred in Londrina, PR (Brazil), during the first semester of 1996. There were included victims from accidents registered by the Military Police, those who died at the accident scenario or on their way to the hospital, those who received emergency care or were hospitalized through the Unified Care System. It was observed a period of 180 days for death verification.
*Baseado na tese de doutorado apresentada à Faculdade de Saúde Pública da USP em 1998.

Pesquisa subvencionada pela Fapesp (Processo no 9700158-9).

Edição subvencionada pela Fapesp (Processo $n^{\circ}$ 00/01601-8).

Recebido em 6/5/1999. Reapresentado em 9/10/1999. Aprovado em 9/1 1/1999. 


\begin{abstract}
Results
The mean incidence and mortality rates were 1,582.2 and 29.0 per 100,000 inhabitants, respectively. These rates, however, have demonstrated great variability in relation to several characteristics (place of residence, gender, age and the victim's mode of transport). The majority of victims were motorcyclists, followed by cyclists and pedestrians who, altogether, accounted for $76.9 \%$ of the total, and $81.5 \%$ of those who died.

Conclusions

These results suggest that road accidents constitute a major cause of morbidity and mortality and that specific preventive measures should be considered in order to reduce such accidents, especially those that involve the most vulnerable road passers.
\end{abstract}

\section{INTRODUÇÃO}

$\mathrm{Na}$ atualidade, os acidentes de transporte terrestre, em especial os de trânsito de veículo a motor, representam, em vários locais do mundo, a principal causa de morte não natural. Nos países desenvolvidos, contudo, essas taxas vêm apresentando valores declinantes, em razão de diversas medidas de prevenção adotadas nessas regiões, ${ }^{9}$ ao contrário das nações mais pobres, onde essas taxas crescem constantemente. ${ }^{10,14}$

Para a área da saúde, os acidentes de transporte terrestre são englobados em um agrupamento de causas de mortes não naturais, as denominadas causas externas, em que se incluem todos os tipos de acidentes (de transporte, quedas, afogamentos e outros), as lesões intencionais (homicídios, suicídios e intervenções legais) e as lesões provocadas em circunstâncias de intencionalidade ignorada (ou causa externa de tipo ignorado), sendo, atualmente, classificadas no Capítulo XX da Classificação Internacional de Doenças, décima revisão. ${ }^{11}$ No Brasil, a importância dessas causas de morte foi sempre crescente, atingindo seu ápice nos anos 80 e, a partir daí, mantendo valores estáveis, porém altos. ${ }^{9}$

Em Londrina, cidade de médio porte localizada ao norte do Estado do Paraná, os acidentes de transporte terrestre são os principais responsáveis pela mortalidade por causas externas (cerca de $40 \%$ desses óbitos) e a primeira causa para os falecidos com idade entre 5 e 39 anos. $^{1}$

Os estudos de mortalidade por causas externas e, especificamente, por acidentes de transporte terrestre, têm fornecido importantes indicações quanto às características das vítimas e quanto à sua magnitude e transcendência em diversas localidades, proporcionando subsídios para a instalação de medidas que visem a reduzir este tipo de morte, geralmente evitável e prematura.
A morte, no entanto, representa apenas a "ponta de iceberg" dos acidentes de transporte terrestre. Sabe-se que, dependendo da localidade, do tipo de acidente e da qualidade da vítima, o número de sobreviventes que demanda cuidados médicos, hospitalização, cuidados de terapia intensiva e caros recursos de apoio diagnóstico pode ser bem maior No entanto, estudos que analisam as vítimas que sobreviveram ao acidente ainda são escassos, principalmente nos países em desenvolvimento. Dessa forma, o presente estudo teve como objetivos verificar a magnitude dos agravos decorrentes de acidentes de transporte terrestre no Município de Londrina e caracterizar, segundo algumas variáveis de importância epidemiológica, as vítimas atendidas em pronto-socorro, internadas e/ou que foram a óbito em conseqüência desses eventos.

\section{MÉTODOS}

A população de estudo foi constituída por 3.643 vítimas de acidentes de transporte terrestre (classificáveis nas rubricas V01 a V89 da Classificação Internacional de Doenças, décima revisão) ${ }^{11}$ referentes a eventos ocorridos no período compreendido entre 1 de janeiro a 30 de junho de 1996 e dentro dos limites geográficos do Município de Londrina. Esse conjunto de vítimas abrangeu as registradas pela Polícia Militar, as que compareceram aos serviços de urgência/emergência conveniados ao Sistema Único de Saúde (SUS) para atendimento médico e/ou internação - para as quais foi observado um prazo de 180 dias, após o acidente, para verificar ocorrência de óbito - e, ainda, aquelas que tenham falecido no local do evento ou no trajeto para o hospital. Os serviços de urgência abrangidos no presente estudo foram todos os 6 hospitais gerais existentes na cidade (4 públicos e 2 filantrópicos) e um privado especializado em ortopedia, sendo excluídos os estabelecimentos com atendimento em outras especialidades. 
Os dados sobre vítimas foram levantados, simultaneamente, pelos boletins de ocorrência da Polícia Militar (BO), das fichas de atendimento de pronto-socorro do SUS, das autorizações de internação hospitalar (AIH) e das declarações de óbito, sendo essas últimas verificadas até 180 dias após a data limite do período de referência de ocorrência dos acidentes. Tais procedimentos permitiram a criação de 4 bancos de dados independentes, os quais foram, posteriormente, cruzados por meio do nome das vítimas e da data do acidente.

Objetivando evitar perda de informações, foram coletados, ainda, dados de pessoas atendidas em pronto-socorro que foram internadas ou que faleceram devido a traumatismo, sem especificação das circunstâncias do evento (causa externa de intenção indeterminada), para posterior verificação se não se tratava de acidente de transporte.

Para as vítimas sem BO buscou-se, por fontes adicionais (comunicações de acidentes de trabalho, notícias em jornais, entrevista com as vítimas ou parentes e verificação de fichas hospitalares), coletar informações que permitissem uma definição, o mais precisa possível, sobre o tipo de acidente, de vítima e do local de ocorrência do acidente.

Após todos os procedimentos adotados para a recuperação de informações importantes para a análise das vítimas, foram excluídas as fichas referentes a vítimas atendidas em prontos-socorros e cujas lesões, tidas inicialmente como devidas a eventos de intenção indeterminada, foram esclarecidas como decorrentes de outros tipos de causas externas (agressões, quedas acidentais, entre outros), de acidente de transporte terrestre, mas ocorrido em outros municípios e, ainda, de acidentes ocorridos anteriormente ao período de referência do estudo.

Durante a fase de pareamento das fichas de atendimento em pronto-socorro com as fichas de acidentes (BO), verificou-se a existência de fichas duplicadas ou triplicadas, na mesma data, referentes à mesma vítima e ao mesmo acidente. Isso deveu-se, segundo informações obtidas junto à instituição de controle das fichas do SUS, ao fato de a vítima ter recebido atendimento de diferentes especialidades médicas, gerando, para cada paciente, duas ou mais fichas na mesma instituição de saúde. Por outro lado, notou-se, também, a existência de vítimas com nomes idênticos e outras que, atendidas em hospital de médio porte, eram, posteriormente, encaminhadas aos hospitais que possuíam Unidades de Terapia Intensiva (UTI) ou especialidades, tendo, portanto, fichas de pronto-socorro em dois hospitais. Dessa maneira, para evitar duplicidade (entrada de uma mesma vítima mais de uma vez no banco de dados), foi realizado controle rigoroso dessas fichas, tanto manual, quanto eletrônico. Foram constatadas, nessa conferência, 209 vítimas com fichas duplicadas por atendimentos em dois diferentes hospitais, as quais foram consideradas como uma única vítima com dois atendimentos. A verificação de homônimos foi realizada tendo como parâmetros a idade e o endereço de moradia, sendo observadas 15 vítimas com homônimos. Houve, ainda, vítimas com dois diferentes acidentes no período de estudo (41 casos), as quais foram consideradas como sendo vítimas de acidentes independentes, sendo computadas, portanto, duas vezes.

Os dados foram apresentados em números absolutos, proporções e coeficientes. Os coeficientes, somente para residentes, foram estimados para o ano completo tendo como base o número de vítimas multiplicado por dois e a população definida na contagem da Fundação IBGE ${ }^{4}$ de 1996, segundo sexo e idade, estimada para 1 de julho.

\section{RESULTADOS}

Do total de 3.643 vítimas de acidentes de transporte terrestre ocorridos no primeiro semestre de 1996, em Londrina, para $3.337(91,6 \%)$ foram localizadas fichas de atendimento em prontos-socorros, $16(0,4 \%)$ faleceram no local do acidente, sendo captadas apenas pelas declarações de óbito, e para 290 (8\%), listadas em BO, não foi possível conhecer sua evolução, pois não foram encontradas fichas de pronto-socorro, de AIH ou de declaração de óbito.

A maioria das vítimas $(92,2 \%)$ foi considerada como de acidente de trânsito (ocorrido em via pública) de veículo a motor, refletindo a alta taxa de motorização da cidade (335,2 veículos por mil habitantes, em 1996). As vítimas restantes resultaram, em grande parte, de acidentes de trânsito de veículo a pedal $(6,5 \%)$.

Quanto ao local de moradia, 3.329 vítimas $(93,7 \%)$ eram residentes no próprio município. $\mathrm{O}$ coeficiente médio de incidência de agravos foi de 1.582,2 por 100.000 habitantes, sendo maior (cerca de $1,5 \mathrm{vez}$ ) entre os residentes da sede urbana em comparação aos residentes nos distritos rurais. Na sede, no entanto, essa taxa variou de 1.266,8 (região centro) até 2.066,1 (região norte), por 100.000 habitantes (Tabela 1).

Motociclistas (condutores ou passageiros) constituíram-se no principal tipo de vítima $(44,4 \%)$, seguidos dos ocupantes de bicicleta $(20,9 \%)$, de carro/caminhonete $(20 \%)$ e pedestres $(11,6 \%)$. Entre as vítimas fatais também prevaleceram os motociclistas $(41,5 \%)$, seguidos dos pedestres $(30,8 \%)$, como mostra a Figura. 
VITIMAS - TOTAL

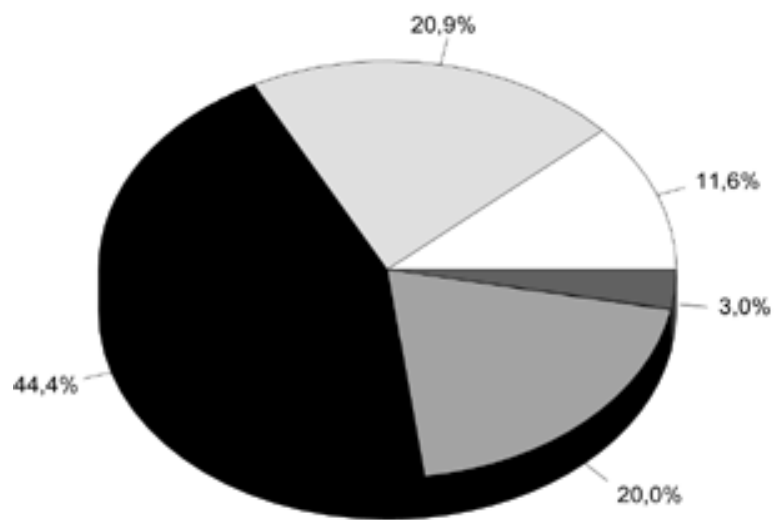

Pedestre
Ciclista

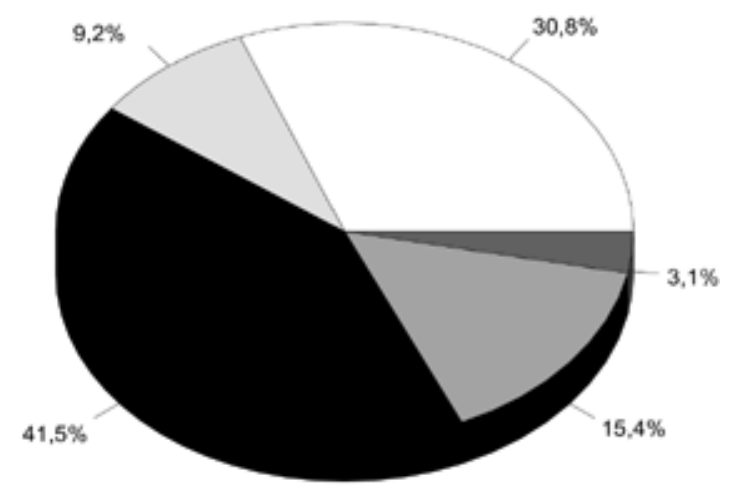

Ocup.carro/caminhonete
Demais

Figura - Vítimas (total e fatais) de acidentes de transporte terrestre ocorridos em Londrina segundo sua qualidade no momento do acidente, 01 de janeiro a 30 de junho de 1996 (\%).

A Tabela 2 mostra alguns indicadores com relação ao sexo e à idade. Os ciclistas apresentaram a menor idade média e mediana $(20,8$ e 17 respectivamente), enquanto pedestres e ocupantes de carro/ caminhonete as maiores. Isso se percebe também no terceiro quartil, que divide os indivíduos ordenados por idade entre os $75 \%$ mais jovens e os $25 \%$ mais idosos: para os pedestres esse valor foi de 44

Tabela 1 - Vítimas de acidentes de transporte terrestre ocorridos em Londrina segundo região de moradia ( $N$, \% e coeficientes de incidência por 100.000 habitantes), 01 de janeiro a 30 de junho de 1996.

\begin{tabular}{lrrr}
\hline Região de moradia & \multicolumn{1}{c}{$\mathrm{N}$} & \multicolumn{1}{c}{$\%$} & Coeficiente \\
\hline Londrina - sede & 3.182 & 95,6 & $1.619,8$ \\
Centro & $(521)$ & $(15,7)$ & $(1.266,8)$ \\
Norte & $(869)$ & $(26,1)$ & $(2.066,1)$ \\
Sul & $(783)$ & $(23,5)$ & $(1.978,3)$ \\
Leste & $(457)$ & $(13,7)$ & $(1.623,0)$ \\
Oeste & $(552)$ & $(16,6)$ & $(1.307,9)$ \\
Londrina - distritos rurais & 147 & 4,4 & $1.052,4$ \\
\hline Total & $3.329 *$ & 100,0 & $1.582,2$ \\
\hline
\end{tabular}

* Somente residentes em Londrina anos e, para ocupantes de carro, 35 anos. Para ciclistas e motociclistas esses valores foram de 27 e 28 anos, respectivamente.

Quanto ao sexo, as menores diferenças entre masculino/feminino (1,6:1 e 1,8:1) foram observadas para ocupantes de carro/caminhonete e pedestres, respectivamente; para ciclistas e motociclistas foram observados os maiores valores $(4,7$ e 3,8 homens para cada mulher).

Com relação à idade, observou-se, ainda, um número considerável de vítimas menores de 18 anos conduzindo veículos automotores. Em análise das 982 vítimas com até essa idade, observou-se que, excluindo as vítimas de acidente de trânsito não especificado (30), 169 $(17,8 \%)$ eram pedestres, $391(41,1 \%)$ ciclistas, 248 motociclistas (26,0\%), 117 ocupantes de carro/caminhonete $(12,3 \%)$ e 27 (2,8\%) ocupantes de outros tipos de veículos. Entre os motociclistas, verificou-se que $96(38,7 \%)$ reconhecidamente conduziam o veículo, sendo que para

Tabela 2 - Vítimas de acidentes de transporte terrestre ocorridos em Londrina segundo sua qualidade no momento do acidente e idades média, desvio-padrão, $1^{\circ}$ quartil, mediana, $3^{\circ}$ quartil e razão masculino/feminino, 01 de janeiro a 30 de junho de 1996.

\begin{tabular}{lcccccc}
\hline & \multicolumn{5}{c}{ Idades (anos) } \\
\cline { 2 - 6 } Qualidade da vítima & Média & $\begin{array}{c}\text { Desvio- } \\
\text { padrão }\end{array}$ & 1ㅇ Quartil & Mediana & 3o Quartil & $\begin{array}{c}\text { Razão } \\
\text { Masc./Fem. }\end{array}$ \\
\hline Pedestre & 28,4 & 22,3 & 9 & 22 & 44 & $1,8: 1$ \\
Ciclista & 20,8 & 12,2 & 13 & 17 & 27 & $4,7: 1$ \\
Motociclista & 24,5 & 8,9 & 19 & 22 & 35 & $3,8: 1$ \\
Ocupante de carro/caminhonete & 28,0 & 14,5 & 19 & 26 & 39 & $2,6: 1$ \\
Ocupante de outros veículos & 28,6 & 15,6 & 17 & 22 & 31 & $2,9: 1$ \\
\hline Total & 25,0 & 13,5 & 17 & 22 & & \\
\hline
\end{tabular}


Tabela 3 - Coeficientes de incidência de agravos e de mortalidade (por 100.000 habitantes) por acidentes de transporte terrestre ocorridos em Londrina segundo qualidade da vítima e faixa etária, 01/01 a 30/06 de 1996.

\begin{tabular}{|c|c|c|c|c|c|c|c|c|c|c|c|c|}
\hline \multirow[b]{3}{*}{ Idade } & \multicolumn{10}{|c|}{ Qualidade de vítima } & & \\
\hline & \multicolumn{2}{|c|}{ Pedestre } & \multicolumn{2}{|c|}{ Ciclista } & \multicolumn{2}{|c|}{ Motociclista } & \multicolumn{2}{|c|}{ Ocup. Auto } & \multicolumn{2}{|c|}{ Demais } & \multicolumn{2}{|c|}{ Total } \\
\hline & Incid. & Mortal. & Incid. & Mortal. & Incid. & Mortal. & Incid. & Mortal. & Incid. & Mortal. & Incid. & Mortal. \\
\hline$<1$ & 26,9 & - & - & - & - & - & 161,1 & - & - & - & 188,0 & - \\
\hline 1 a 4 & 318,2 & 6,6 & 119,3 & - & 106,1 & - & 165,7 & - & 39,8 & 6,6 & 755,7 & 13,3 \\
\hline 5 а 9 & 254,9 & - & 362,0 & - & 51,0 & - & 107,1 & - & 5,1 & - & 790,2 & - \\
\hline 10 a 14 & 212,3 & 4,8 & 801,0 & 4,8 & 202,7 & - & 149,6 & 4,8 & 67,6 & - & $1.462,0$ & 14,5 \\
\hline 15 a 19 & 141,5 & - & 825,1 & - & $1.744,6$ & 33,0 & 424,4 & 18,9 & 47,2 & - & $3.305,3$ & 51,9 \\
\hline 20 a 24 & 140,7 & 5,4 & 384,3 & - & $2.398,0$ & 37,9 & 595,4 & 10,8 & 65,0 & - & $3.653,9$ & 54,1 \\
\hline 25 a 29 & 103,9 & - & 311,7 & - & $1.443,9$ & 38,3 & 607,1 & 5,5 & 49,2 & - & $2.570,6$ & 43,8 \\
\hline 30 а 39 & 108,8 & 11,8 & 223,5 & 5,9 & 550,0 & 2,9 & 288,2 & - & 64,7 & - & $1.273,5$ & 20,6 \\
\hline 40 a 49 & 150,4 & 11,9 & 186,0 & 4,0 & 265,1 & - & 229,5 & 4,0 & 51,4 & - & 918,1 & 19,8 \\
\hline 50 a 59 & 214,4 & 24,5 & 73,5 & 12,2 & 165,4 & 12,2 & 177,6 & - & 42,9 & 6,1 & 722,7 & 55,1 \\
\hline 60 a 69 & 188,2 & 28,2 & 47,1 & - & 47,1 & - & 131,8 & 9,4 & 18,8 & - & 432,9 & 37,6 \\
\hline 70 a 79 & 335,3 & 39,4 & 59,2 & - & 19,7 & - & 138,1 & - & - & - & 572,0 & 39,4 \\
\hline $80 \mathrm{e}+$ & 320,5 & - & - & - & - & - & - & - & 53,4 & - & 373,9 & - \\
\hline Total & 178,7 & 9,0 & 335,5 & 2,9 & 689,1 & 11,4 & 290,4 & 4,8 & 46,6 & 1,0 & $1.582,2$ & 29,0 \\
\hline
\end{tabular}

Tabela 4 - Vítimas de acidentes de transporte terrestre ocorridos em Londrina segundo sua qualidade no momento do acidente e taxas de internação e coeficiente de letalidade (\%).

\begin{tabular}{|c|c|c|c|c|c|}
\hline \multirow[b]{2}{*}{ Qualidade da vítima } & \multicolumn{3}{|c|}{ Internados } & \multicolumn{2}{|r|}{ Óbitos } \\
\hline & $\mathrm{N}$ total & N & Taxa* & $\mathrm{N}$ & Coef. de Letalidade* \\
\hline Pedestre & 411 & 74 & 18,0 & 20 & 4,9 \\
\hline Ciclista & 738 & 57 & 7,7 & 6 & 0,8 \\
\hline Motociclista & 1.570 & 183 & 11,7 & 27 & 1,7 \\
\hline Ocupante de carro/caminhonete & 707 & 57 & 8,1 & 10 & 1,4 \\
\hline Ocupante de caminhão & 31 & 4 & 12,9 & 1 & 3,2 \\
\hline Ocupante de ônibus & 54 & 5 & 9,3 & - & - \\
\hline Pessoa montada em animal & 17 & - & - & - & - \\
\hline Ocupante de trator & 5 & 1 & 20,0 & 1 & 20,0 \\
\hline Não especificada & 110 & 12 & 10,9 & - & - \\
\hline Total & 3.643 & 393 & 10,8 & 65 & 1,8 \\
\hline
\end{tabular}

* \% em relação ao total de vítimas em cada categoria

26,6\% desses menores a posição no veículo era ignorada. Entre os ocupantes de carro/caminhonete a proporção de condutores foi de apenas $2,6 \%$, sendo, no entanto, desconhecida a posição de $10,3 \%$.

Homens se acidentaram principalmente como condutores de veículos $(88,2 \%$ dos motociclistas e $61,9 \%$ dos ocupantes de carro/caminhonete do sexo masculino), enquanto mulheres predominaram como passageiras $(51,8 \%$ das motociclistas e $76,2 \%$ das ocupantes de carro/caminhonete).

A Tabela 3 apresenta os coeficientes de incidência de agravos e de mortalidade segundo faixa etária e qualidade da vítima no momento do evento. Nas faixas etárias de 50 a 59 anos e de 15 a 24 anos foram observados os maiores coeficientes de mortalidade, superiores a 50 por 100.000 habitantes. Analisando esses coeficientes por faixa etária e qualidade da vítima, os valores mais elevados foram observados para jovens motociclistas de 15 a 29 anos, com taxas de 33 a 38,3 por 100.000 habitantes, e para idosos pedestres, com coeficientes de 28,2 (60 a 69 anos) e 39,4 (70 a 79 anos).
No geral, motociclistas de 20 a 24 anos e de 15 a 19 anos apresentaram os maiores coeficientes de incidência de agravos resultantes de acidentes de transporte terrestre (2.398 e $1.744,6$ por 100.000 habitantes, respectivamente). De 1 a 4 anos, o maior coeficiente de incidência foi apresentado por pedestres; de 5 a 14 por ciclistas; de 15 a 49 por motociclistas e, a partir dos 50 anos, por pedestres.

O coeficiente médio de letalidade foi de $1,8 \%$ e a taxa de internação de $10,8 \%$ (Tabela 4), com variações importantes de acordo com a qualidade da vítima no momento do acidente. Desconsiderando ocupantes de caminhão e de trator, em razão do pequeno número de casos, os maiores coeficientes de letalidade e de proporção de pacientes internados foram verificados entre pedestres $(4,9 \%$ e $18 \%$, respectivamente) e motociclistas (1,7\% e 11,7\%).

Das 65 vítimas que faleceram em um intervalo de observação de 180 dias após o acidente, $16(24,6 \%)$ morreram no local do evento, 27 (41,5\%) no prontosocorro e $22(33,8 \%)$ após internação. Ao final da primeira semana, $92,3 \%$ dos óbitos já haviam ocorri- 
do e, ao final de um mês, 96,9\%. O intervalo máximo verificado entre o acidente e o óbito foi de 64 dias.

\section{DISCUSSÃO}

O presente trabalho buscou, pelas diversas fontes de informação disponíveis, levantar a totalidade de vítimas (fatais ou não) de acidentes de transporte terrestre ocorridos em Londrina, em um período determinado de tempo. Ainda assim, é possível que uma pequena parcela de vítimas sobreviventes ao acidente possa não ter sido captada, por motivos diversos (atendimento por meio de planos privados de saúde, encaminhamento a hospitais de cidades vizinhas ou por não terem procurado assistência médica em nível de pronto-socorro). Essa última suposição é corroborada pelo fato de não terem sido localizadas fichas de pronto-socorro para 290 vítimas listadas em BO policial. Por outro lado, optou-se por trabalhar com o local de ocorrência do acidente, em vez de residência das vítimas, devido à sua maior utilidade para as áreas de planejamento e de administração de tráfego, em qualquer contexto. Por esses motivos, de ordem metodológica, os valores quanto à incidência de agravos e de mortalidade por acidentes de transporte terrestre, apresentados nessa investigação, devem ser considerados como "mínimos".

Apesar disso, o coeficiente médio de incidência de agravos por esses acidentes foi extremamente alto (1.582,2 por 100.000 habitantes), bem como o coeficiente de mortalidade (29 por 100.000 habitantes), sendo esse composto, em sua quase totalidade, por acidentes de trânsito de veículo a motor (28 por 100.000 habitantes), superior ao apresentado por muitas capitais brasileiras. ${ }^{9}$ Entre os casos fatais, a análise do tempo entre o acidente e o óbito sugere que esses acidentes são graves, pois apenas 33,8\% das vítimas faleceram após internação e, em uma semana, $92,3 \%$ das mortes já haviam ocorrido.

Os coeficientes de incidência desagregados por região da cidade evidenciaram diferentes riscos de seus habitantes sofrerem agravos decorrentes desses acidentes, sendo maiores para as regiões norte e sul da sede urbana, o que pode estar relacionado a diferentes exposições a situações de tráfego. Grande parte dessas duas regiões é composta por conjuntos habitacionais populares, o que faz supor, caso seus habitantes envolvam-se em acidentes, que sejam, na maioria, pedestres ou usuários de veículos de preço mais acessível (como bicicleta e motocicleta) e menos seguros para os ocupantes, na eventualidade de acidentes.

Por outro lado, como salientaram Capistrano Filho \& Rumel, ${ }^{2}$ interesses especulativos levaram os con- juntos habitacionais de várias cidades para as periferias, muitas vezes próximos a rodovias. Em Londrina, essa realidade não é diferente, com duas rodovias (uma federal e outra estadual) separando as regiões norte e sul da região central. As populações dessas duas áreas, evidentemente, estão expostas a um maior risco de sofrerem acidentes mais graves, devido ao tamanho dos veículos de carga que trafegam diariamente nessas vias e às altas velocidades possíveis de serem adotadas em alguns trechos.

Quanto ao perfil por sexo e idade, os resultados são semelhantes a diversos estudos que indicam pessoas do sexo masculino e jovens como os tipos de vítimas mais freqüentes, ${ }^{5,7,9,10}$ ainda que os coeficientes de mortalidade sejam, geralmente, mais altos entre idosos. ${ }^{5,13}$ Esse perfil é conseqüência, provavelmente, da maior exposição masculina e de jovens no trânsito e por comportamentos determinados social e culturalmente, que os fazem assumir maiores riscos na condução de veículos, como maior velocidade, manobras mais arriscadas, uso de álcool, entre outros.

Verificou-se que as mulheres, como vítimas, ainda estiveram, predominantemente, na posição de passageiras, ao contrário dos homens, que se traumatizaram principalmente como condutores, o que reflete normas socialmente aceitas, em nosso meio, do homem assumir a condução do veículo na maioria das situações. É possível, entretanto, que esse quadro se altere em futuro próximo, por estar ocorrendo, nos últimos anos, uma clara tendência de aumento da participação feminina na condução de veículos automotores, devido à sua crescente inserção no mercado de trabalho, maior independência e conseqüente maior exposição ao tráfego das cidades. ${ }^{5,16}$

Número considerável de menores de idade foi vítima ao dirigir veículos automotores, principalmente motocicletas. Ressalta Koizumi ${ }^{7}$ que dificilmente um menor de idade teria condições financeiras para adquirir uma moto, cabendo aos pais a determinação de quando e como esse menor vai utilizar esse veículo. Nem sempre, porém, essa determinação é consonante com a legislação vigente e acompanhada de orientação e educação no que se refere às práticas a serem observadas na condução segura de veículos nas vias públicas. Em trabalho desenvolvido em Marília, interior do Estado de São Paulo, Dellatorre ${ }^{3}$ constatou que adolescentes de classes sociais média e alta aprenderam a dirigir precocemente, com idades variando de oito a treze anos, tendo como instrutores membros da própria família. Esses dados indicam ser necessário o estabelecimento de estratégias que coíbam esse tipo de comportamento no cotidiano brasi- 
leiro, o qual, indubitavelmente, aumenta o risco de ocorrência de acidentes. ${ }^{12}$

O conhecimento quanto ao papel desempenhado pela vítima no momento do acidente é fundamental para a implantação de estratégias preventivas. Essa identificação foi facilitada a partir do uso, em 1996, da Classificação Internacional de Doenças, décima revisão (CID10), para codificação das causas de morte no Brasil, ${ }^{8}$ a qual foi utilizada para a classificação das vítimas no presente trabalho. Esse procedimento permitiu verificar que os motociclistas representaram o principal tipo de vítima, tanto entre o total $(44,4 \%)$, como entre as que faleceram (41,5\%). O uso de motocicletas tem sido crescente no Brasil, por ser uma opção mais econômica, comparada a outros veículos automotores, além das facilidades de tráfego e estacionamento. Nesse contexto, tem sido observado, nos últimos anos, um aumento de sua utilização como instrumento de trabalho na entrega de mercadorias, medicamentos, alimentos ou documentos e, até mesmo, no transporte de passageiros. Seus condutores (os chamados "motoboys" ou "mototaxistas") estão constantemente expostos à possibilidade de ocorrência de acidentes, seja por sua maior exposição nas vias públicas, seja por realização de manobras arriscadas ou por velocidades adotadas com vistas à realização rápida de tarefas e conseqüente aumento da produtividade.

Motociclistas, juntamente com pedestres e ciclistas, caracterizam-se por constituírem o grupo de usuários da via pública mais vulnerável em termos de exposição corpórea a lesões em caso de acidentes. No presente estudo, esse conjunto de vítimas representou $76,9 \%$ do total e $81,5 \%$ das fatais. Resultado semelhante foi encontrado em estudo realizado em Maringá, cidade também localizada ao norte do Paraná, onde $81 \%$ das vítimas que morreram por acidentes de trânsito eram motociclistas, ciclistas ou pedestres..$^{13}$ Nesse citado estudo argumenta-se, com propriedade, que esse padrão pode ser comum a várias cidades do interior, principalmente onde há predomínio de dias ensolarados e temperaturas ambientes elevadas, como é o caso de Londrina. Nessas circunstâncias, bicicletas e motocicle- tas constituem-se em meio comum de transporte, principalmente para alguns grupos sociais que não podem arcar com os custos de aquisição e de manutenção de veículos mais seguros.

Pedestres, por outro lado, representaram as vítimas com maior probabilidade de serem internadas ou morrerem após terem sido traumatizados em um acidente ( $18 \%$ e $4,9 \%$, respectivamente). Gawryszewski, ${ }^{5}$ a esse respeito, cita apropriadamente publicação da Companhia de Engenharia de Tráfego (CET) de São Paulo, referindo que "o atropelamento é um choque entre $800 \mathrm{Kg}$ de aço, em velocidade, contra $79 \mathrm{Kg}$ de carne e osso. O pedestre tem poucas chances". A análise dos coeficientes de incidência e de mortalidade, para esse tipo de vítima, no presente estudo, indicou como mais expostos ao risco de serem vítimas, as crianças e os idosos, o que pressupõe que ações de prevenção desses acidentes devam levar em conta as características e as limitações próprias dessas fases da vida, com compensações positivas no meio ambiente, tais como criação de espaços seguros para o lazer de crianças, estratégias que reduzam a velocidade dos veículos, faixas/passarelas exclusivas para pedestres e semáforos em pontos estratégicos. ${ }^{6,15}$

Em última análise, os achados do presente estudo retratam peculiaridades de vítimas de acidentes de transporte terrestre ocorridos em um município de médio porte, que apontam necessidades de intervenção específicas, principalmente para a redução desses eventos quando envolvem pedestres, ciclistas e motociclistas. A fragilidade e a predominância desses usuários da via pública como vítimas reforçam argumentos já intensamente colocados sobre a necessidade de intervenções amplas, intersetoriais e multiprofissionais, que promovam um ambiente mais favorável para a circulação dessas pessoas. ${ }^{2,3,5,9,13-15}$ Nesse sentido, há que se reavaliar as políticas públicas atuais de estímulo ao transporte individual em automóveis, com pouca ênfase na melhoria do ambiente para a circulação mais segura e eficiente de pedestres e outros tipos de veículos, como bicicletas e ônibus de transporte coletivo público.

\section{REFERÊNCIAS}

1. Andrade SM, Martins HFC, Melchior R. Perfil de mortalidade de residentes em Londrina, 1996. Londrina: Departamento de Informações em Saúde; 1997.

2. Capistrano Filho D, Rumel D. Acidentes de trânsito, problema de saúde pública. In: Capistrano Filho D, Pimenta AL, organizadores. Saúde para todos: um desafio ao município: a resposta de Bauru. São Paulo: HUCITEC; 1988. p. 86-91.

3. Dellatorre MCC. O trânsito e seus novos centauros [dissertação]. Londrina: Centro de Ciências da Saúde da Universidade Estadual de Londrina; 1997.

4. Fundação IBGE. Departamento Regional Sul. Divisão de Pesquisa do Paraná. Contagem da população 1996: dados distritais - população por situação e sexo. Rio de Janeiro; s/d. 
5. Gawryszewski VP. A mortalidade por causas externas no Município de São Paulo, 1991 [dissertação]. São Paulo: Faculdade de Saúde Pública da USP; 1995.

6. Hoxie RE, Rubenstein LZ. Are older pedestrians allowed enough time to cross intersections safely? J Am Geriatr Soc 1994;42:241-4.

7. Koizumi MS. Padrão das lesões nas vítimas de acidentes de motocicleta. Rev Saúde Pública 1991;26:306-15.

8. Laurenti R. Acidentes e violências/lesões e envenenamentos e a $10^{a}$ revisão da Classificação Internacional de Doenças. Rev Saúde Pública 1997;31(4 Supl):55-8.

9. Mello Jorge MHP, Gawryszewski VP, Latorre MRDO. Acidentes e violências no Brasil. I - Análise dos dados de mortalidade. Rev Saúde Pública 1997;31(4 Supl):5-25.

10. Odero W, Garner P, Zwi A. Road traffic injuries in developing countries: a comprehensive review of epidemiological studies. Trop Med Int Health 1997;2:445-60.
11. Organização Mundial da Saúde. Manual de classificação estatística internacional de doenças e problemas relacionados à saúde. 10a rev. São Paulo: Centro Colaborador da OMS para Classificação de Doenças em Português; 1993. v.1.

12. Reeder Al, Chalmers DJ, Langley JD. Rider training, reasons for riding, and the social context of riding among young on-road motorcyclists in New Zealand. Aust NZ J Public Health 1996;20:369-74.

13. Scalassara MB, Souza RKT, Soares DFPP. Características da mortalidade por acidentes de trânsito em localidade da região Sul do Brasil. Rev Saúde Pública 1998;32:125-32.

14. Söderlund N, Zwi AB. Traffic-related mortality in industrialized and less developed countries. Bull World Health Organ 1995;73:175-82.

15. Stevenson M. Childhood pedestrian injuries: what can changes to the road environment achieve? Aust NZ Public Health 1997;21:33-7.

16. Waters C, Gibbons L, Semenciw R, Mao Y. Motor vehicle traffic accidents in Canada, 1978-87 by time of occurrence. Rev Can Santé Publique 1993;84:58-9. 